\title{
Modelling of Two-Component Turbulent Mass and Heat Transfer in Air-Fed Pressurised Suits
}

\author{
Krzysztof Tesch • Michael W. Collins • \\ Tassos G. Karayiannis • Mark A. Atherton • \\ Paul Edwards
}

Received: 7 January 2010 / Accepted: 21 January 2011 / Published online: 1 February 2011

(C) The Author(s) 2011. This article is published with open access at Springerlink.com

\begin{abstract}
In this paper the modelling of an important industrial problem is addressed, which involves the two-component turbulent flow with heat transfer that takes place inside protective clothing. The geometry of the flow boundaries is reconstructed in a CAD system from photogrammetry scan data. The overall model is sufficiently realistic to allow, after validation, design improvements to be tested. Those presented here allow the reduction of hotspots over the worker's body surface and increase thermal comfort.
\end{abstract}

Keywords Two-component turbulence $\cdot$ Heat transfer Protective suit

\section{Nomenclature}

$\begin{array}{ll}c, c_{v} & \text { specific heat capacity }\left[\mathrm{kJ} \mathrm{kg}^{-1} \mathrm{~K}^{-1}\right] \\ C & \text { heat lost by the skin by convection and conduction }\left[\mathrm{Wm}^{-2}\right] \\ C_{\varepsilon i}, C_{\mu}, C_{i} & \text { turbulence model constants } \\ \boldsymbol{D} & \text { strain rate tensor }\left[\mathrm{s}^{-1}\right] \\ D & \text { diffusivity }\left[\mathrm{m}^{2} \mathrm{~s}^{-1}\right]\end{array}$

K. Tesch $(\bowtie)$

Fluid Mechanics Department, Gdańsk University of Technology,

ul. G. Narutowicza 11/12, 80-233 Gdańsk, Poland

e-mail: krzyte@pg.gda.pl

M. W. Collins · T. G. Karayiannis - M. A. Atherton

School of Engineering and Design, Brunel University,

Middlesex UB8 3PH, Uxbridge, UK

P. Edwards

Culham Science Centre, UKAEA Culham Division,

Oxfordshire OX14 3DB, Abingdon, UK 


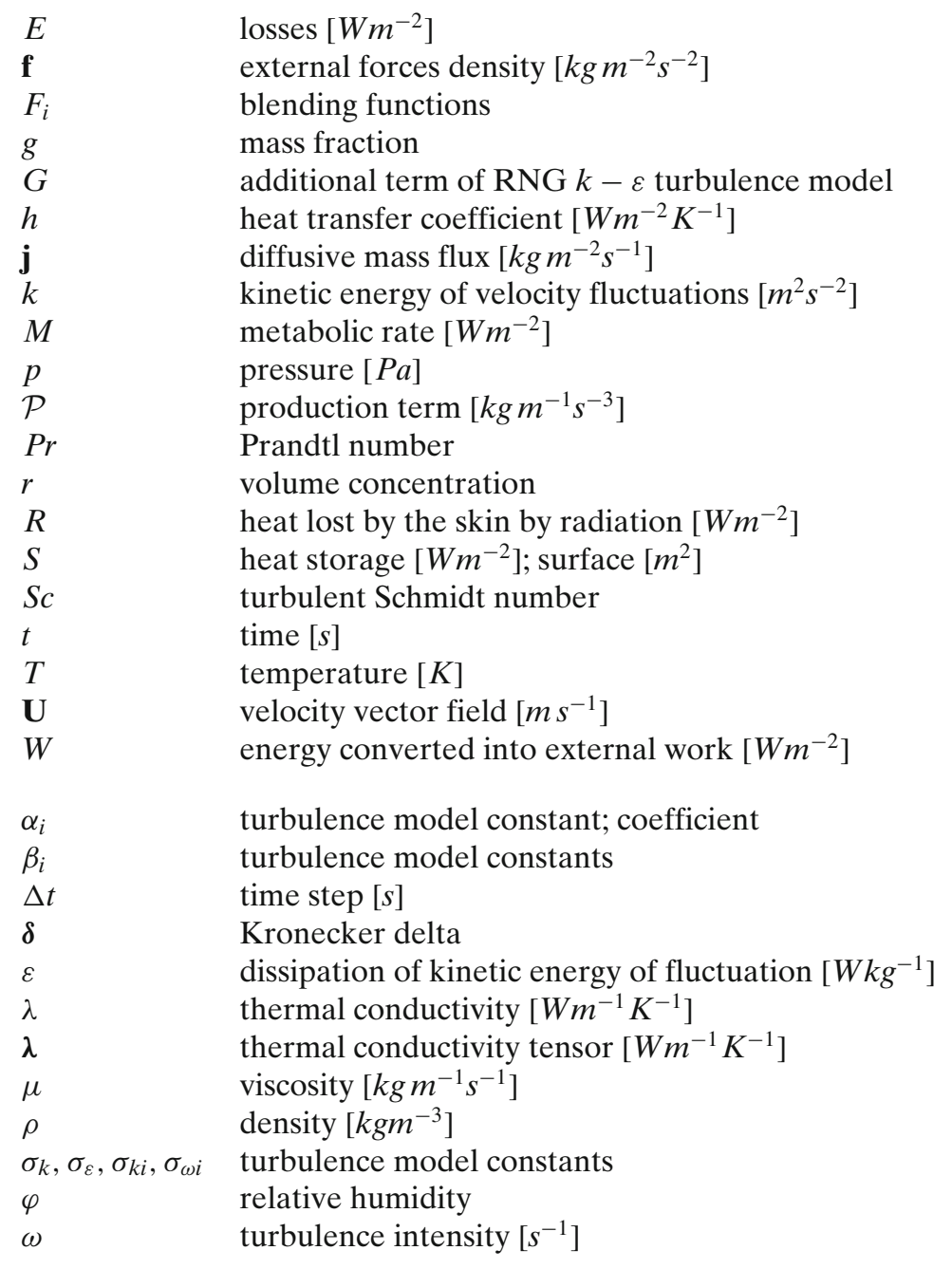

Superscripts

+
$i$
$\overline{(})$

dimensionless

individual species

averaged

$\begin{array}{ll}\text { Subscripts } & \\ a & \text { ambient } \\ b & \text { body } \\ d & \text { dew } \\ e & \text { effective } \\ h & \text { head } \\ r & \text { respiratory } \\ s & \text { saturated, surface, evaporative } \\ t & \text { turbulent }\end{array}$




\section{Introduction}

The JET (Joint European Torus) fusion reactor at Culham, near Oxford, UK is an integral part of the international fusion programme. During reactor shutdown periods it is necessary to clean up and adapt the internal surfaces of the reactor torus, much of which relies on manual labour. Air-fed pressurised suits are used by workers to protect them against radioactive contamination, which includes airborne tritium and surface beryllium. A pressurised air supply is needed to minimise inward leakage of tritium through the suit material. The multi-layer suit is also designed to prevent, as far as possible, accidental damage which would cause additional leakage. Substantial cooling of the air supply is needed because workers using the suits have to operate in thermal comfort conditions if they are to be effective. More recently, health and safety legislation has highlighted the need for a comprehensive model able to address all thermo-fluids aspects of the performance of the worker-suit combination [1]. Such a model needs to demonstrate quantitative reliability.

This quantitative reliability is achieved by a detailed study of the threedimensional turbulent flow within the microclimate, which is defined as the space between the suit and the worker. This space can be time-varying due to workers' actions and movements. The microclimate flow is complex, therefore, in a number of aspects. The geometric complexity was addressed by an industrial-scale experiment involving the positioning of a movable mannequin with and without an actual suit. Using sophisticated photogrammetry and three-dimensional reconstruction it is possible to determine accurately both the suit and mannequin geometries and hence that of the microclimate itself.

Because air moisture content is a key feature of a workers' state of thermal comfort, the thermo-fluids problem itself required the modelling of both heat and mass transfer under two-component conditions. In this paper we report a model, Fig. 1, using basic analysis for these conditions. This model also utilised various

Fig. 1 Diagrammatic explanation of model

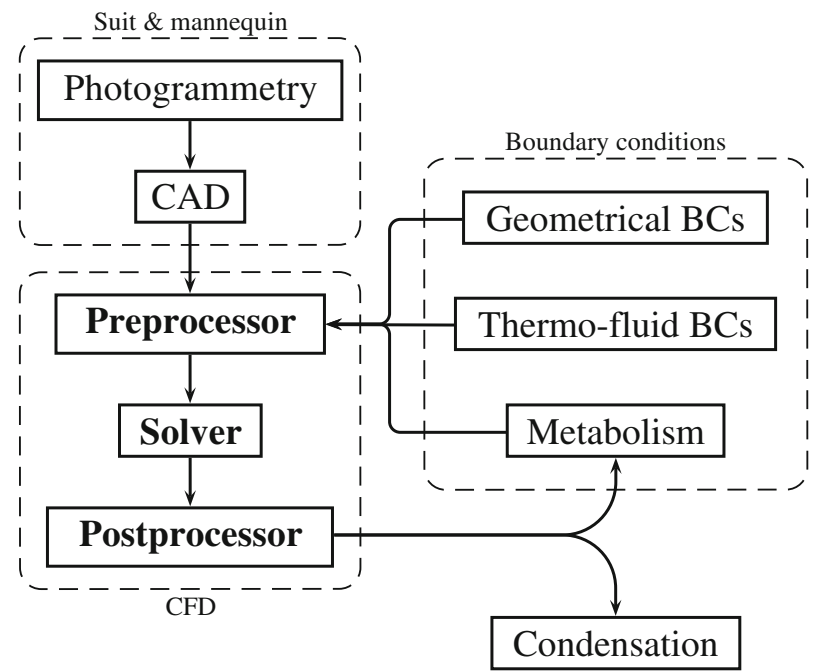


two-equation turbulence models. The model comprised: diffusive heat transfer using Fourier's law, mass transfer using Fick's law, and a two-component model resulting from the mixing of air and water vapour. The vapour is present because of moisture produced by the human body as well as that generated by respiration. The twocomponent flow makes it also possible to predict surface condensation on a suit. The flow itself is transient, not only because of workers' movements, but also by virtue of the respiratory cycle and because of variations in the worker's task-dependent metabolism. The latter is responsible for variations in body and surface temperatures under different human working loads. Further experiments, entailing suited workers, permitted validation comparisons and are presented here.

The detailed programme of predictions firstly involved the generation of sets of data for the closest cases in the literature, those of flows round unsheathed and sheathed cylinders. The latter constitute a 'clothed limb'. The predictions included comparisons using all turbulence models available in the software and these, too, are reported here.

\section{Modelling Two-Component Flow}

\subsection{General equations}

As developed here the two component equations derived from the summation of the two-phase equations $[8,10,12]$. Individual species transport equations may be written as

$$
\frac{\partial\left(\rho g^{i}\right)}{\partial t}+\nabla \cdot\left(\rho g^{i} \mathbf{U}\right)=-\nabla \cdot \mathbf{j}^{i}
$$

The functions and values of component $i$ are denoted by superscript $i$. The symbol $g^{i}$ denotes mass fraction, the mixture density $\rho$ is defined as the sum of individual species densities $\rho^{i}$ and volume concentration and the velocity of the mixture is represented by the sum of individual species velocities and mass fractions. The diffusive mass flux $\mathbf{j}^{i}$ is the mass transfer among species. This transfer requires further modelling. The sum of the above equation gives the mass conservation equation in the same form as that for single-component flow.

The momentum conservation equation for a two-component flow takes the following form

$$
\frac{\partial(\rho \mathbf{U})}{\partial t}+\nabla \cdot(\rho \mathbf{U U})=\rho \mathbf{f}+\nabla \cdot \boldsymbol{\sigma}-\nabla \cdot \sum_{i=1}^{2} \rho g^{i} \tilde{\mathbf{U}}^{i} \tilde{\mathbf{U}}^{i}
$$

where $\rho \mathbf{f}=\sum_{i=1}^{2} \rho^{i} g^{i} \mathbf{f}^{i}$ is the density of external forces. This equation derives from the summation of the two-phase equations. The mixture stress tensor $\sigma=\sum_{i=1}^{2} r^{i} \sigma^{i}$, where the individual stress tensor is generalised to $\boldsymbol{\sigma}^{i}=-p^{i} \boldsymbol{\delta}+2 \mu^{i} \boldsymbol{D}^{D i}+\mu_{v}^{i} \boldsymbol{\delta} \nabla \cdot \mathbf{U}^{i}$. In the above equation $p^{i}$ is the pressure, $\mu^{i}$ and $\mu_{v}^{i}$ are the molecular and bulk viscosity respectively, $\boldsymbol{D}^{D i}$ the strain rate tensor deviator and $\delta$ the Kronecker delta. An additional term should be noted, which is not present in the single-component version of this equation. This term is responsible for diffusion of the momentum. 
The inner energy transport equation or Fourier-Kirchhoff equation for a mixture takes the following form

$$
\frac{\partial(\rho e)}{\partial t}+\nabla \cdot(\rho \mathbf{U} e)=\phi_{\mu}-\nabla \cdot \mathbf{q}-\nabla \cdot \sum_{i=1}^{2} e^{i} \mathbf{j}^{i}
$$

The heat vector of a mixture is denoted here as $\mathbf{q}=\sum_{i=1}^{2} r^{i} \mathbf{q}^{i}$. The additional term in the above equation is due to internal energy diffusion resulting from a concentration difference.

Not taking compressibility effects into account makes it is possible to simplify the above equations. The density is assumed to be constant. Additionally, if the diffusive velocity is small compared with the mixture velocity it can be assumed that all the fluid properties $\varphi^{i}$ except the concentration (mass or volume fraction) share the same fields $\varphi^{i}=\varphi$. This means that the velocity, pressure and temperature fields are shared by all the components. The mass and momentum conservation equations for the incompressible mixture take the form equivalent to those for the single-component case. To obtain this, it is assumed that all the second order terms involving diffusive velocity may be neglected. If the diffusive velocity is small then its product is even smaller. The constitutive equation for individual stress tensors also simplifies to its single-component version.

The heat vector is described here by means of Fourier's law in the form of $\mathbf{q}=$ $-\lambda \cdot \nabla T$. And it is another constitutive equation for anisotropic fluids. For isotropic fluids the conductivity tensor $\lambda$ may be expressed in terms of the isotropic part $\lambda=$ $\lambda \delta$. Here the symbol $\lambda$ represents the thermal conductivity coefficient and Fourier's law simplifies to

$$
\mathbf{q}=-\lambda \nabla T
$$

Mass transport behaves similarly to heat transfer. Fick's law expresses the mass flux stream in the same manner as Fourier's law for a heat flux, namely $\mathbf{j}^{i}=$ $-\rho \boldsymbol{D}^{i j} \cdot \nabla g^{i}$. The diffusivity tensor $\boldsymbol{D}^{i j}$ is usually replaced by the kinematic diffusivity coefficient $D^{i j}$, giving Fick's law as

$$
\mathbf{j}^{i}=-\rho D^{i j} \nabla g^{i}
$$

More detailed discussion may be found in [17].

\subsection{Averaged equations}

Assuming that the density is constant we have the average form of the mass conservation equation

$$
\nabla \cdot \overline{\mathbf{U}}=0
$$

The Reynolds equation is of the same form as for single-component flow

$$
\rho \frac{\partial \overline{\mathbf{U}}}{\partial t}+\rho \nabla \cdot(\overline{\mathbf{U}} \overline{\mathbf{U}})=\rho \overline{\mathbf{f}}-\nabla \bar{p}_{e}+\nabla \cdot\left(2 \mu_{t} \overline{\boldsymbol{D}}\right)
$$


where effective pressure $p_{e}:=\bar{p}+2 / 3 \rho k$ and effective viscosity is composed of eddy and molecular viscosity $\mu_{e}:=\mu_{t}+\mu$. The averaged concentration transport equation may be obtained by means of the eddy diffusivity hypothesis

$$
\frac{\partial \bar{g}^{i}}{\partial t}+\nabla \cdot\left(\bar{g}^{i} \overline{\mathbf{U}}\right)=\nabla \cdot\left(D_{e} \nabla \bar{g}^{i}\right)
$$

The effective diffusivity may be represented as a function of the eddy viscosity and the turbulent Schmidt number $D_{e}:=\mu_{t} \rho^{-1} S c_{t}^{-1}+D^{i j}$. We have two species, namely air and water vapour. Yet there is no need to solve another differential equation. This is because all the species concentration have to sum to unity. Another algebraic relation is given, namely the constraint equation in the form of

$$
\bar{g}^{2}=1-\bar{g}^{1} .
$$

The averaged Fourier-Kirchhoff equations takes the form

$$
\rho c_{v}\left(\frac{\partial \bar{T}}{\partial t}+\nabla \cdot(\bar{T} \overline{\mathbf{U}})\right)=2 \mu \overline{\boldsymbol{D}}^{2}+\nabla \cdot\left(\lambda_{e} \nabla \bar{T}+\rho \bar{T} \sum_{i=1}^{2} c_{v}^{i} D^{i j} \nabla \bar{g}^{i}\right)+\rho \varepsilon
$$

The correlation of the temperature and the concentration fluctuation are neglected. The effective conductivity $\lambda_{e}$ arises from the eddy diffusivity hypothesis. It can be expressed using the turbulent Prandtl number $\operatorname{Pr}_{t}$, as $\lambda_{e}:=\mu_{t} c_{v} \operatorname{Pr}_{t}^{-1}+\lambda$.

The additional two equations depend on the two-equation turbulence model. The production term is defined as $\mathcal{P}:=2 \mu_{t} \overline{\boldsymbol{D}}: \overline{\boldsymbol{D}}$. The four turbulence models considered were:

(i) $k-\varepsilon$ [9]. Two additional transport equations are those for modelled kinetic energy of velocity fluctuation $k$ which comes from the Reynolds stress transport equation

$$
\rho \frac{d k}{d t}=\mathcal{P}+\nabla \cdot\left(\left(\frac{\mu_{t}}{\sigma_{k}}+\mu\right) \nabla k\right)-\rho \varepsilon
$$

and dissipation $\varepsilon$ of kinetic energy of fluctuation which is analogous to $k$ transport

$$
\rho \frac{d \varepsilon}{d t}=C_{\varepsilon 1} \frac{\varepsilon}{k} \mathcal{P}+\nabla \cdot\left(\left(\frac{\mu_{t}}{\sigma_{\varepsilon}}+\mu\right) \nabla \varepsilon\right)-C_{\varepsilon 2} \rho \frac{\varepsilon^{2}}{k} .
$$

The eddy viscosity is defined as $\mu_{t}=C_{\mu} \rho k^{2} \varepsilon^{-1}$. The five constants in the above equations should be deduced from experiment for a specific geometry. This 'standard' set is given by $\sigma_{k}=1, \sigma_{\varepsilon}=1.3, C_{\mu}=0.09, C_{\varepsilon 1}=1.44, C_{\varepsilon 2}=1.92$.

(ii) RNG $k-\varepsilon$ [20]. The renormalisation group (RNG) method makes it possible to express the problem by means of large scale and time equations. Applying RNG methods on the Navier-Stokes equations allows their use on a coarser mesh. The equation for kinetic energy $k$ transport has almost the same form as for the standard $k-\varepsilon$ model

$$
\rho \frac{d k}{d t}=\mathcal{P}+\nabla \cdot\left(\frac{\mu_{e}}{\sigma_{k}} \nabla k\right)-\rho \varepsilon .
$$


The only difference is the effective viscosity $\mu_{e} \sigma_{k}^{-1}$ instead of $\mu_{t} \sigma_{k}^{-1}+\mu$. The same concerns the dissipation transport equation where also a new term $G$ appears

$$
\rho \frac{d \varepsilon}{d t}=C_{\varepsilon 1} \frac{\varepsilon}{k} \mathcal{P}+\nabla \cdot\left(\frac{\mu_{e}}{\sigma_{\varepsilon}} \nabla \varepsilon\right)-C_{\varepsilon 2} \rho \frac{\varepsilon^{2}}{k}-G .
$$

The 'standard' set of constants differs from that for the $k-\varepsilon$ model, i.e. $\sigma_{k}=$ $1, \sigma_{\varepsilon}=1.3, C_{\mu}=0.085, C_{\varepsilon 1}=1.42, C_{\varepsilon 2}=1.68$. Effective viscosity definition remains either the same or is redefined by $\mu_{e}:=\mu\left(1+\mu_{t} \mu^{-1}\right)^{2}$.

(iii) $k-\omega[19]$. The turbulent frequency $\omega$ is proportional to the ratio of dissipation and kinetic energy $\varepsilon=C_{\mu} k \omega$. The eddy viscosity takes the following form $\mu_{t}=$ $\rho k \omega^{-1}$. The transport equation for $k$

$$
\rho \frac{d k}{d t}=\mathcal{P}+\nabla \cdot\left(\left(\frac{\mu_{t}}{\sigma_{k 1}}+\mu\right) \nabla k\right)-C_{\mu} \rho k \omega .
$$

The transport equation for $\omega$

$$
\rho \frac{d \omega}{d t}=\alpha_{1} \frac{\omega}{k} \mathcal{P}+\nabla \cdot\left(\left(\frac{\mu_{t}}{\sigma_{\omega 1}}+\mu\right) \nabla \omega\right)-\beta_{1} \rho \omega^{2} .
$$

The 'standard' set of constants $\sigma_{k 1}=2, \sigma_{\omega 1}=2, C_{\mu}=0.09, \alpha_{1}=5 / 9, \beta_{1}=$ $3 / 40$.

(iv) SST [11]. The shear stress transport model combines the $k-\omega$ near the wall and the $k-\varepsilon$ far from it

$$
\begin{aligned}
& \rho \frac{d k}{d t}=\mathcal{P}+\nabla \cdot\left(\left(\frac{\mu_{t}}{\sigma_{k 3}}+\mu\right) \nabla k\right)-C_{\mu} \rho k \omega, \\
& \rho \frac{d \omega}{d t}=\alpha_{3} \frac{\omega}{k} \mathcal{P}+\nabla \cdot\left(\left(\frac{\mu_{t}}{\sigma_{\omega 3}}+\mu\right) \nabla \omega\right)-\beta_{3} \rho \omega^{2}+\left(1-F_{1}\right) 2 \frac{\rho}{\omega} \sigma_{\omega 3} \nabla k \cdot \nabla \omega .
\end{aligned}
$$

Constants marked with subscript ' 3 ', namely $\sigma_{k 3}, \sigma_{\omega 3}, \alpha_{3}, \beta_{3}$ are linear combinations of combined models constants $C_{3}=F_{1} C_{1}+\left(1-F_{1}\right) C_{2}$. The SST turbulence model redefines eddy viscosity to avoid over-prediction of shear stresses near the wall $\mu_{t}=\rho a_{1} k \max ^{-1}\left(a_{1} \omega, S F_{2}\right)$. Invariant measure of strain rate $S$ and blending functions $F_{1}, F_{2}$ may be found in [11].

In terms of numerical modelling of two component flow one has to deal with the averaged additional individual species transport equation. This is because the rest of the variables such as velocity, pressure and temperature share the same fields. The species transport equation has the form of a general transport equation and may be treated numerically in a standard way as described in [4] for instance.

All the turbulence models considered predict the outlet temperature from the suit wall [15]. The differences between single-and two-component model results, however, are found to be negligible. This is true in terms of the outlet temperature at least and may be explained by the small concentration of the vapour. The averaged mass fraction of vapour leaving the suit was $\bar{g}^{2}=0.0132 \%$, whereas the same fraction of exhaled air was $\bar{g}^{2}=2.37 \%$. The two-component flow is important if one wishes to predict occurrence of surface condensation. It occurs when the surface temperature 
Fig. 2 Surface and dew temperatures difference on face [15]

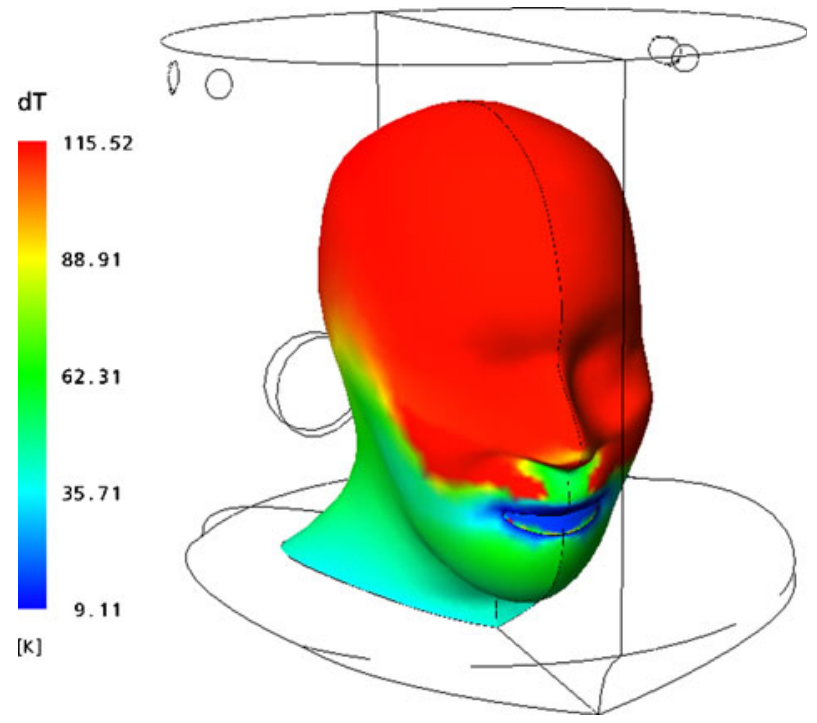

$T_{s}$ is lower than the dew point temperature $T_{d}$. The dew point temperature may be found as a function of pressure and temperature by means of the empirical formula [13]

$$
T_{d}=C_{0}+\sum_{i=1}^{4} C_{i} \alpha^{i-1}+C_{5} e^{0.1984 \alpha} .
$$

In this formula, the coefficient $\alpha$ is defined as $\alpha:=\ln \left(p_{s} \varphi\right)$ and the set of constants is given by $C_{0}=273, C_{1}=6.54, C_{2}=14.426, C_{3}=0.7389, C_{4}=0.09486, C_{5}=0.4569$. The product of saturated vapour pressure $p_{s}$ and relative humidity $\varphi$ may be expressed as a function of pressure and vapour concentration by means of the following equation:

$$
p_{s} \varphi=\frac{\bar{g}^{2} p}{0.622\left(1-\bar{g}^{2}\right)+\bar{g}^{2}} .
$$

This method is only able to predict occurrence of condensation. It does not take account of other phenomena such as local change of geometry or thermal properties.

Figure 2 shows a typical set of differences between surface and dew temperatures $d T:=T_{s}-T_{d}$. Had surface condensation occurred this difference $d t$ would have been negative. However, neither prediction nor experiment showed such phenomena.

\section{Modelling of Geometry}

The purpose of this reconstruction was to produce a CAD model of a pressurised suit in a variety of positions for use in thermo-fluid simulations. This was achieved by clothing a full size 'marching' mannequin in a real suit, as shown in Fig. 3. The mannequin was supplied by DSTL (Defence Science and Technology Laboratories, 
Fig. 3 Mannequin in a suit

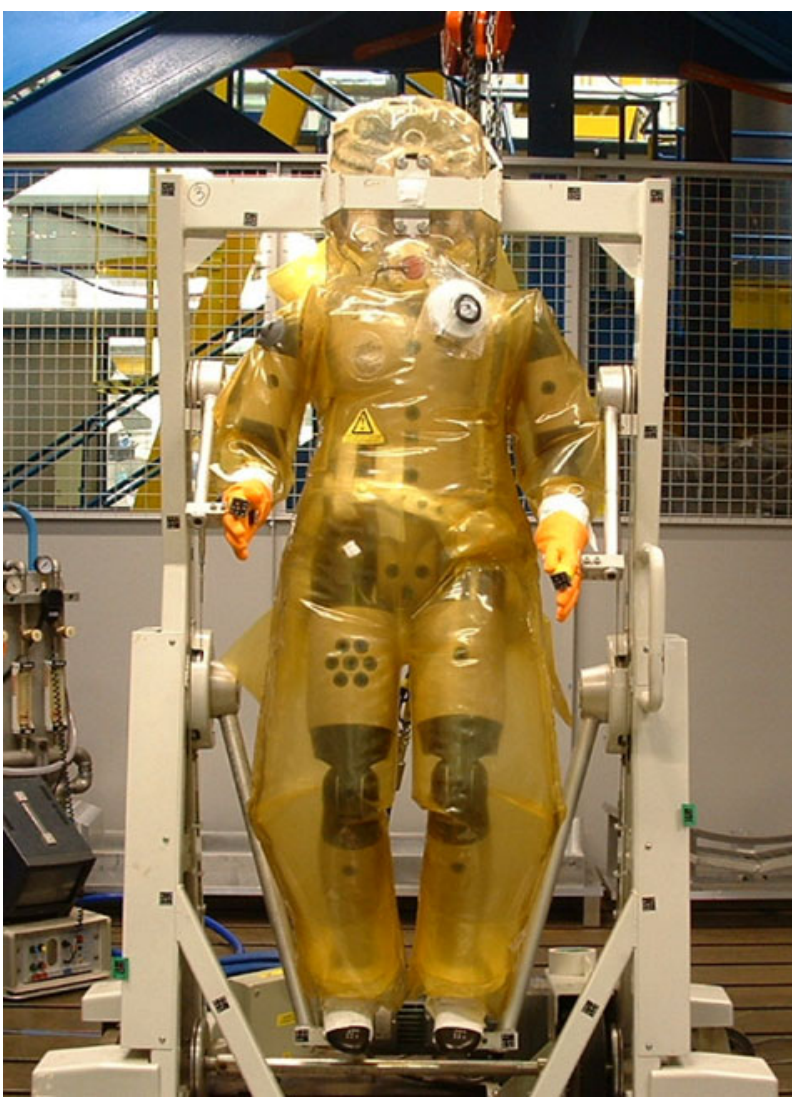

Porton Down, U.K.) to UKAEA for this purpose, and the latter's existing capabilities for scanning and inspection allowed the production of a points cloud data set, typically as may be seen in Fig. 4. For more details see [2]. The mannequin in Fig. 3 is a static, dry, isothermal facility, yet it can give valuable information. There are also more advanced construction such as that described in [3].

The scanned data was obtained by photogrammetry, a remote sensing technology in which geometric properties of objects are determined from photographic images. Many photographs of the object are taken from different angles allowing common points to be identified on each image. A line of sight can then be constructed from the camera location to a point on the object. It is the intersection of these rays that determines the three-dimensional location of the point. The scanned data was then processed semi-automatically to obtain the CAD model of Fig. 4. It should be appreciated that this process is both demanding and time-consuming. For example, some fine details may be lost during this process because certain points are missing and one has to extrapolate the shape of the cross section. Additionally, some noise and random measurement errors were present and had to be smoothed out. It should be noted that the feet and palms are absent due to the use of close-fitting glove cuffs and boots. Hence no flow occurs beyond the geometry indicated in Fig. 4 because these regions do not form part of the flow domain, i.e. physically, they experience 
Fig. 4 Cloud points (left) and the reconstructed geometry (right)
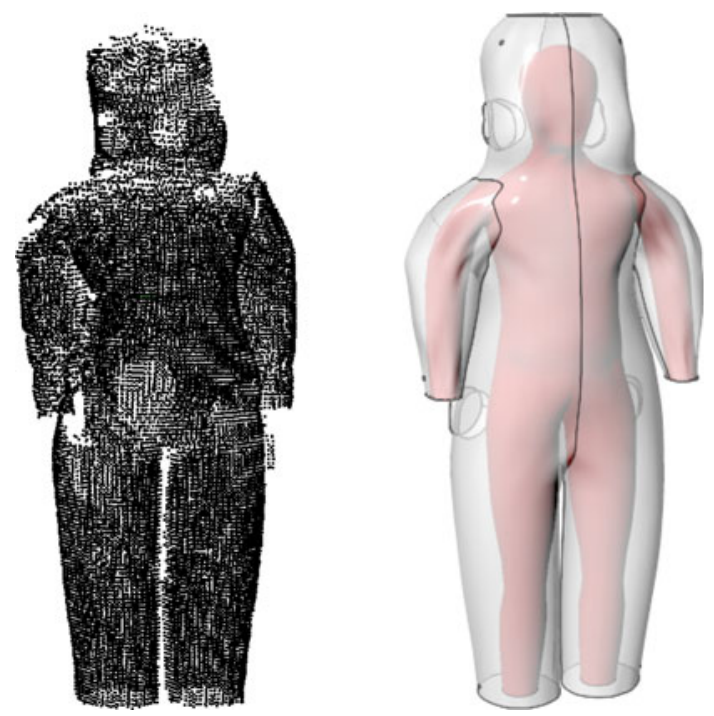

no flow. This significantly reduces the demands of the geometry reconstruction, as there is no need to reconstruct, for example, the shapes of fingers, which are very complicated and would need extra computer resources for discretisation.

\section{Boundary Conditions}

The boundary conditions are:

(i) Inlet. The suit presented in Fig. 5 has six inlets: two each above the head, the wrists and near the ankles. The volumetric flow rate of $390 \mathrm{l} / \mathrm{min}$ at $290.7 \mathrm{~K}$ has been uniformly divided between the six inlets. The inlet Reynolds number was $R e \approx 10^{4}$. Instead of $k, \varepsilon$ or $\omega$ the turbulence intensity $\tau_{t}$ and the viscosity ratio $\mu_{t} / \mu$ are specified. The specified values refers to low intensity. Even for low intensity heat transfer is intensified in comparison with laminar flows. This is advantageous from the cooling point of view.

(ii) Outlet. There are four outlets in Fig. 5: two below the head (where the outlet temperatures were measured) and two at the lower part of the back. Constant static pressure has been specified there.

(iii) Symmetry. Because of the symmetry of the geometry only half of the computational domain has been taken into consideration. This means that all the scalar values $\varphi$ must fulfil $\frac{\partial \varphi}{\partial n}=0$. However, it should be borne in mind that arm or leg movements would remove this symmetry, requiring the entire worker/suit combination to be addressed. Some asymmetrical geometries are presented in [16].

(iv) Wall. All the velocity components equal zero. The bulk heat flux $q_{n}$ is specified by means of the overall heat transfer coefficient $h$ and ambient temperature $T_{a}$. Finding or calibrating the value of heat transfer coefficient $h$ is not a trivial task and a detailed discussion of this may be found in [14]. The convective heat 
Fig. 5 Original geometry of pressurised suit
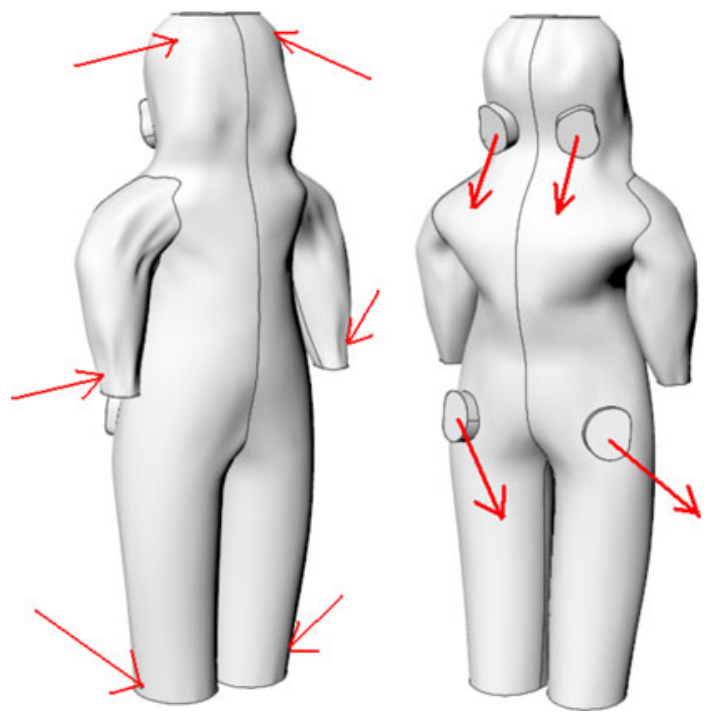

transfer mode is the most common but it is also the least easy to determine because it depends on all the parameters controlling the fluid field including surface characteristics and thermal properties of the fluid. Determining the heat transfer coefficient is the goal of predictive and experimental investigations for a given problem. The calibration problem was to find a heat transfer coefficient value $h$ that minimised either one or two objectives in a fitness function using a specialised procedure involving evolutionary algorithms [18]. Individual functions for calibration (i.e. minimisation of errors) were defined as differences between measurements and numerical prediction of the temperatures at the suit outlets. The single minimised function was formulated as a difference of the two individual errors. This means that minimisation of such a function leads to an optimal solution based on equal (i.e. balanced) errors. Figure 6 shows the distribution of such a function. The optimal/calibrated value of the heat transfer coefficient was found to be $h \approx 10 \mathrm{Wm}^{-2} \mathrm{~K}^{-1}$. More sophisticated minimisation procedures are also possible [14].

For the ambient side of the suit the temperature equals $T_{a}=292 \mathrm{~K}$. At the body side the situation is much more complex. Among other factors the body temperature depends on metabolic rate (activity), environment and clothing. Additionally, there is the question of person to person variation. There is a constant (balanced) heat transfer between the human body and environment due to convection, conduction and radiation. The simplest method of calculating body temperature variation relies on the accepted concept of thermal balance, discussed in detail in [17]. This makes it possible to predict a time-dependent surface temperature as a consequence of the workers' metabolic processes that affect it. The body temperature variation $T_{b}$ may then be described by means of the ordinary differential equation, (see derivation in [17])

$$
\frac{d T_{b}}{d t}+\frac{A_{d} h}{c m} T_{b}=\frac{A_{d}}{c m}\left(M-W+h T-E_{s}-E_{r}\right),
$$


Fig. 6 Difference between measured and predicted temperatures $f$ as a function of $h$ [14]

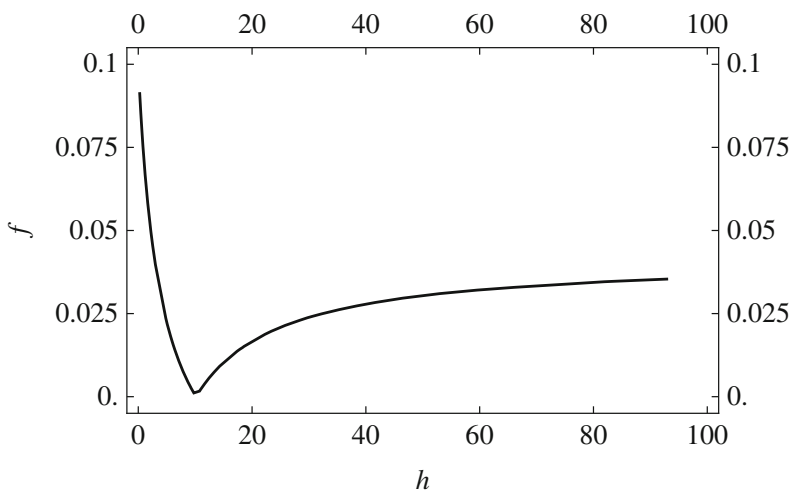

where $A_{d}$ stands for body surface, $c$ body specific heat, $m$ body mass, $M$ metabolic rate, $W$ energy converted into external work, $E_{r}, E_{s}$ respiratory and evaporative losses. The above Eq. 21 arises from the human thermal balance condition mentioned previously. The condition may be written as [5-7]

$$
S=M-W-R-C-E_{r}-E_{s} .
$$

Here $S$ stands for heat storage, $C$ and $R$ for heat lost by the skin by convection plus conduction and radiation respectively. Various empirical formulae for $M$, $W, E_{r}$ and $E_{s}$ are accessible in the literature. A sample solution of Eq. 21 is shown in Fig. 7 for a typical test case relevant to a JET shutdown procedure. The first 36 min represents a stabilising period where no physical activity took place. From 36 to 49 min a moderate walking activity is assumed. During the period from 49 to $68 \mathrm{~min}$ a hard activity-working is undertaken. The final period of $68-82$ min corresponds to metabolic cooling without any physical activity. Because of the heat capacity of the body accommodated in Eq. 21 there is a time-lag of temperature when the metabolic load changes, as shown in Fig. 7.

Fig. 7 Predicted body temperature variation

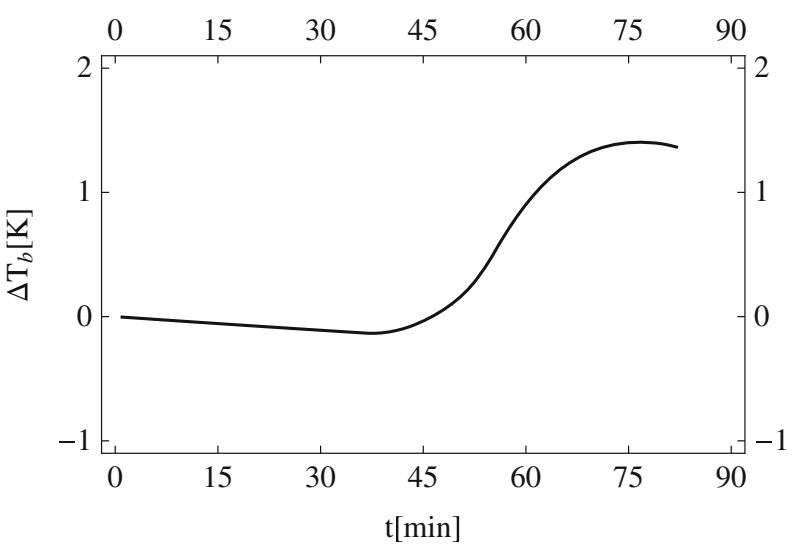


Fig. 8 Outlet temperature predictions compared with measured data

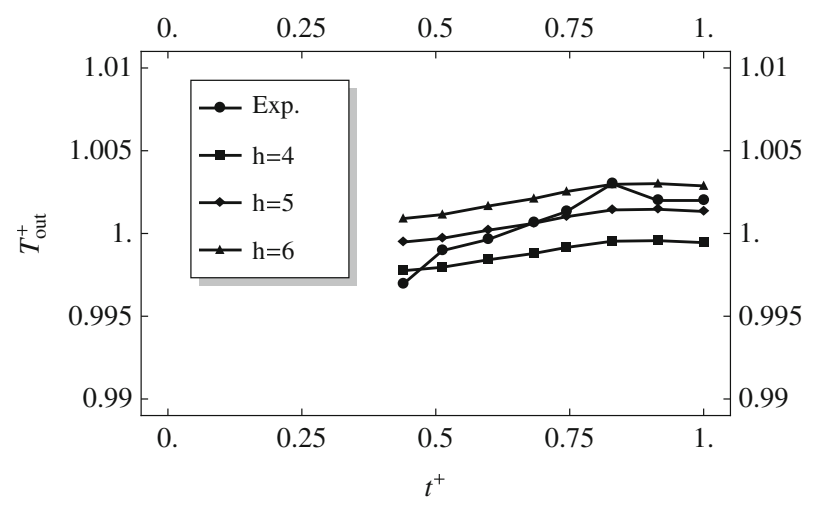

\section{Accuracy Aspects: Comparisons with Experimental Data and Mesh Sensitivity Checks}

Figure 8 gives a comparison between experimental data for suit outlet temperatures and CFD predictions using the standard turbulence $k-\varepsilon$ model. The dimensionless temperatures $T^{+}$are defined as $T^{+}:=T / T_{a}$ and dimensionless time as $t^{+}:=t / \Delta t$, where $\Delta t$ stands for total measurement time. Experimental data were collected in the upper outlet, as shown by the topmost downward-facing arrows in Fig. 5. More specifically, Fig. 8 presents the transient variation of outlet temperature as a function of body side heat transfer coefficient $h\left[W^{-2} \mathrm{~K}^{-1}\right]$. This comparison suggests that the heat transfer coefficient is not constant but varies at least as a function of temperature. If so, $h$ would effectively vary with time and fit the experimental data better.

Figures 9 and 10 show similar comparisons to Fig. 8, but for front and rear torso temperatures. The predictions were performed for three different heat transfer coefficients (from the body side), namely 5, 6,7 $\mathrm{Wm}^{-2} \mathrm{~K}^{-1}$ to fit the experimental data. Quite good agreement (except for the peaks) can be seen. The only other discernible difference is in the the cooling period where experimental values decrease more rapidly than predictions.

Fig. 9 Front torso temperature variation

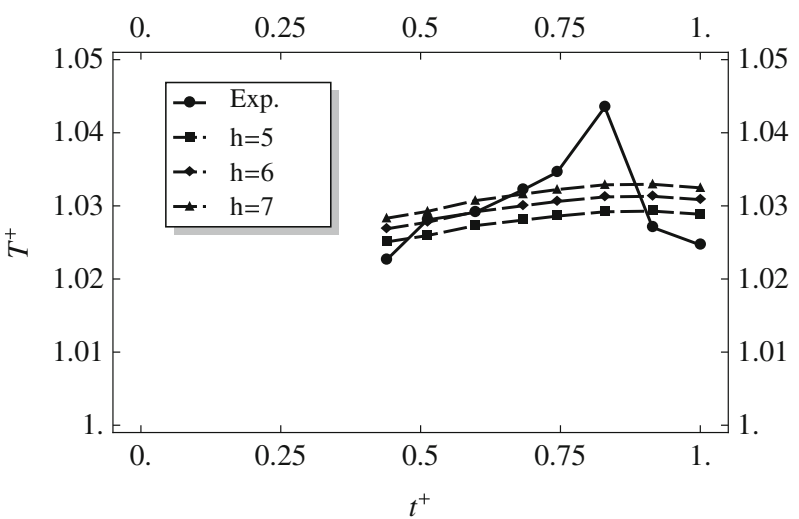


Fig. 10 Rear torso temperature variation

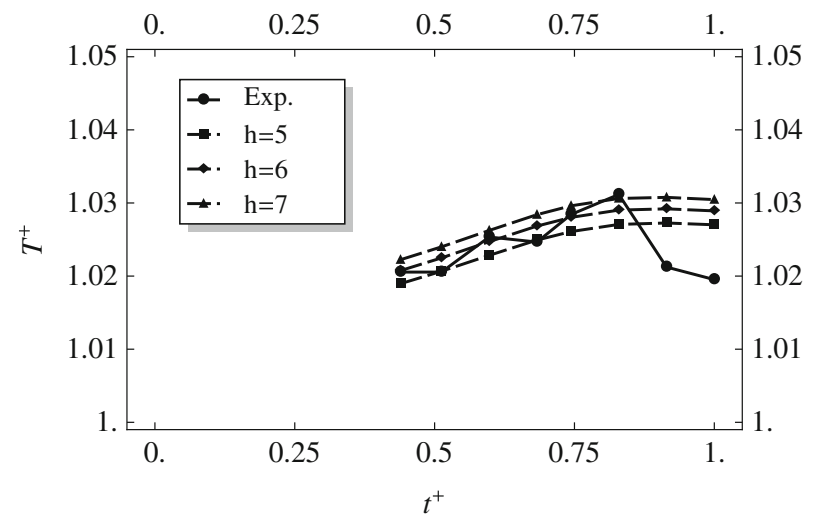

There are several reasons for the differences between measurements and predictions. Firstly the geometry of the human in the experiments is different than the scanned mannequin data used in predictions. While the calculations are transient they are steady state in terms of flow domain configuration (geometry). Observational experience indicates that there may be phenomena that influence temperature distribution such as the 'pumping' effect due to workers' movements, namely walking, bending, and other arm and leg motions. It is difficult to perform hard work in a steady position and this means calculations which are steady state

Fig. 11 Discretised geometry of the mannequin
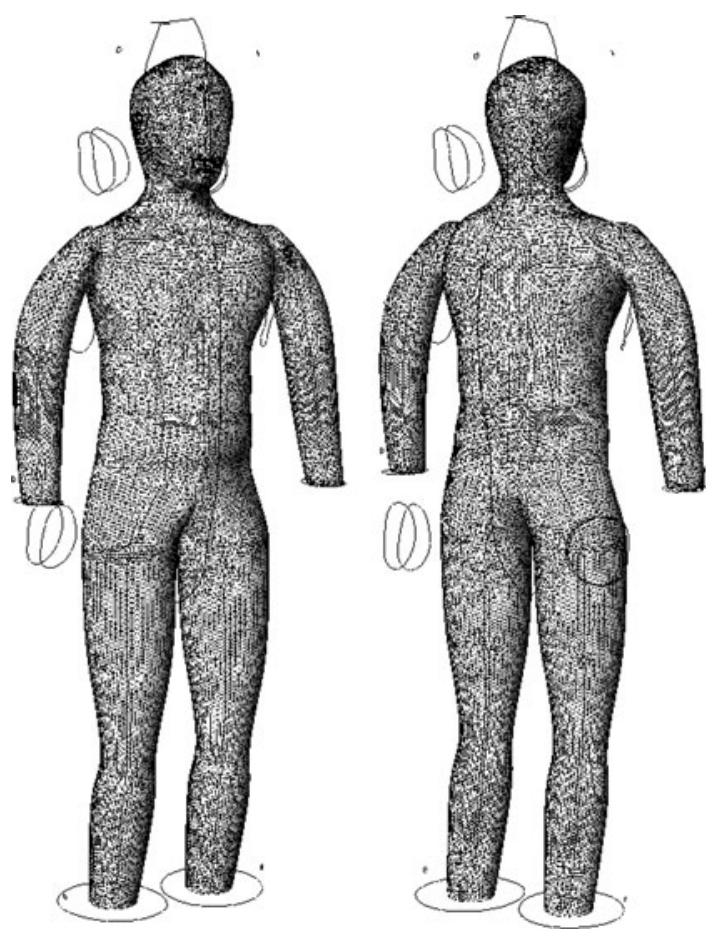
in terms of configurations do not reflect experimental reality. Measurements were taken in 'stand up' position just after the resting and cooling periods. In the meantime the person was seated. This would cause a part of the back of the suit to be blocked, meaning an absence of cooling air to the corresponding part of the body. However, there was no alternative to this human experimental procedure. It is not practical for the worker to be kept in the same standing position for $30 \mathrm{~min}$ to ensure stabilisation. For predictions, the heat transfer coefficient was assumed to be constant, that is independent of local (small-scale) suit/body configuration or temperature. For the latter this definitely seems to be untrue. Comparative predictions have shown that at lower temperatures better agreement is obtained using lower values of $h$, whereas for higher temperatures higher $h$ values are better. This suggests that including a temperature function of $h$ would improve the correlation. Not unrelated to this is the assumption in the current study that the metabolic model is zero-dimensional: the core temperature is assumed to be constant in the whole body. This affects any calculation of human surface temperature. For the remaining calculations of our study we used a constant value of $h=5 \mathrm{Wm}^{-2} \mathrm{~K}^{-1}$.

\section{Gridding and Mesh Sensitivity}

If the reconstructed geometry from Fig. 4 is sufficiently accurate it can then be discretised. The discretised surface of the mannequin is presented in Fig. 11 and the surface of the suit itself in Fig. 12. The whole process requires considerable care and was carried out manually. It took between two and three full working weeks to achieve a successfully discretised geometry from the cloud point data. While it is

Fig. 12 Discretised geometry of the suit
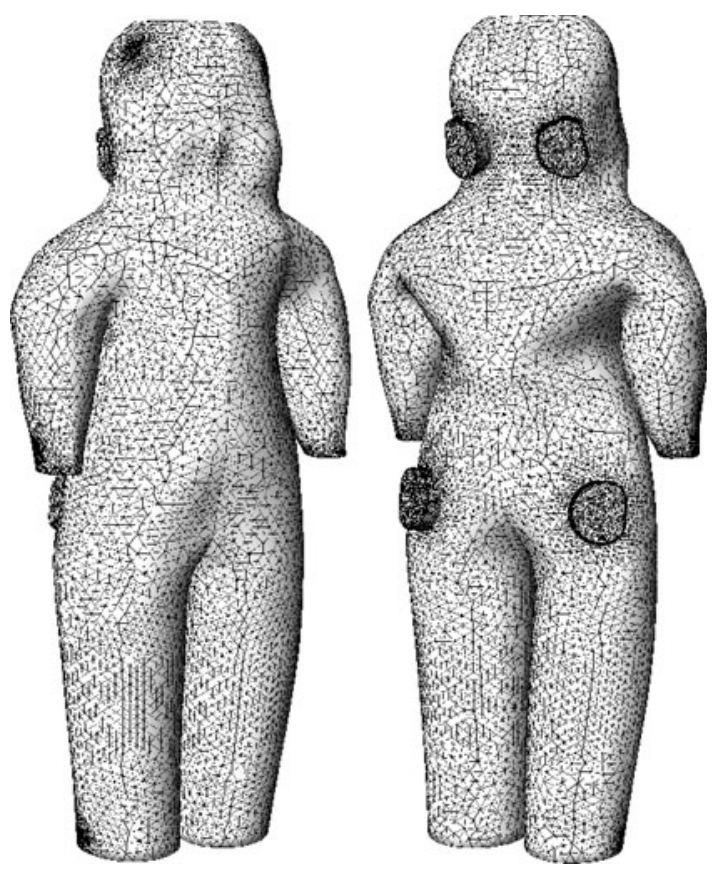
Table 1 Mesh statistics

\begin{tabular}{lr}
\hline & Number \\
\hline Nodes & 272,224 \\
Elements total & 775,033 \\
Tetrahedral & 392,878 \\
Wedges & 374,588 \\
Pyramids & 7,567 \\
\hline
\end{tabular}

possible to automate some part of the process it could not be guaranteed that the reconstructed geometry would be good enough for the CFD preprocessor software to mesh. There is also the problem of missing data in heavily concave surfaces. These data must be reconstructed to be consistent with the adjacent curvature of the suit geometry. Such a process demands direct operator input and judgement. These qualities are also essential where the reverse problems exist. At positions such as the joint between arm and torso there are valid data points that cannot be easily attributed to specific parts of the body and suit.

Table 1 shows the mesh statistics for the reconstructed geometry in Figs. 11 and 12. In this case, that of a standing mannequin, only half of the geometry needed to be considered due to its symmetry.

Figure 13 demonstrates mesh sensitivity by showing the influence of inflation size (number of elements) on the outlet temperature. By 'inflation' it is meant the increase in number of elements such as wedges and pyramids as opposed to triangular elements at the surface. An inflated mesh is more computationally efficient around the boundaries where the velocity gradients are very high. Increasing or decreasing the number of elements by about \pm 0.1 million of the mesh (with this inflation) has negligible effects on the outlet temperatures. This is partly because the total number of wedges is still high. The situation is slightly different where there is no inflation. One can observe little influence of the mesh size and slightly less agreement between measurements and CFD prediction compared with an inflated mesh. Results are nearly constant within the range of $0.7-0.9$ million of elements. Predictions are slightly less accurate compared with the inflated mesh. Also, it takes a longer time to reduce the computational error to the same level for the same mesh size as the inflated grid. The only benefit of the non-inflated mesh is lower memory demand.

Fig. 13 Mesh sensitivity

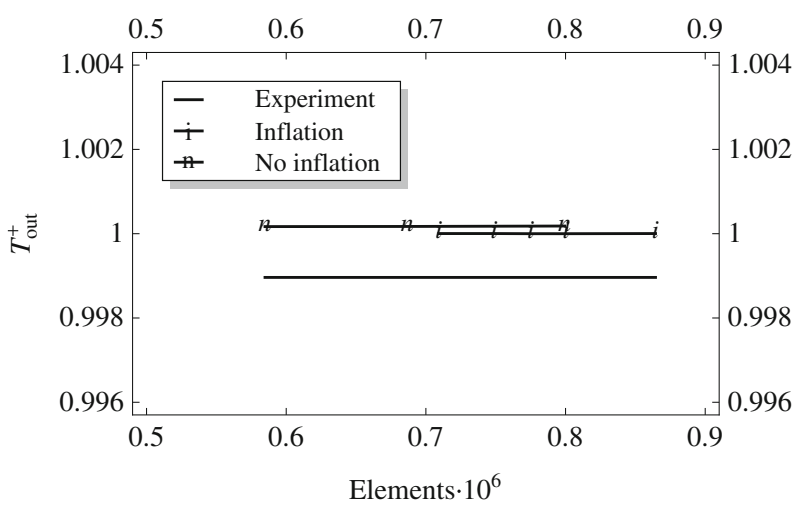




\section{Design Improvement}

Having such a generic and validated model enables us to perform meaningful computational experiments for any working conditions and over the entire parametric range of operating data. The ultimate objective is thereby to propose design improvements. In Section 7.1 we report the results of a series of predictions based on the original design and configuration of the suit as shown in Fig. 5. These studies should answer important user questions about possible real problem situations that could occur when using the current design of suit. Such could be where either arm, leg or head inlets are blocked. For example, it happens frequently that the leg inlets are blocked by long boots. This means that the rest of the mass flow rate is divided among the other inlets equally. However, another user experience is that the mass flow rate is not divided equally among all the inlets. These studies then enable us to make proposals (Section 7.2) for modifying the suit to allow greater advantage to be taken of the cooling air capacity.

\subsection{Effects of blockage situations on suit performance}

In this study the concept of total body temperature is used. Firstly dimensionless temperatures $T^{+}$are defined by means of surface average and ambient temperatures

$$
T^{+}:=\frac{1}{T_{a}|S|} \iint_{S} T d S,
$$

where $S$ stands for surface. The total body temperature $T_{b}^{+}$is composed of head $T_{h}^{+}$ and body $T_{t}^{+}$(head excluded) contributions by means of the following definition

$$
T_{b}^{+}:=\frac{\left|S_{h}\right|}{\left|S_{b}\right|} T_{h}^{+}+\frac{\left|S_{t}\right|}{\left|S_{b}\right|} T_{t}^{+}
$$

Using the definition of standard case (the situation when air is divided among all the inlets equally) the case of blocked leg inlets is firstly considered. It can be

Fig. 14 Dimensionless temperatures - standard vs blocked leg inlets

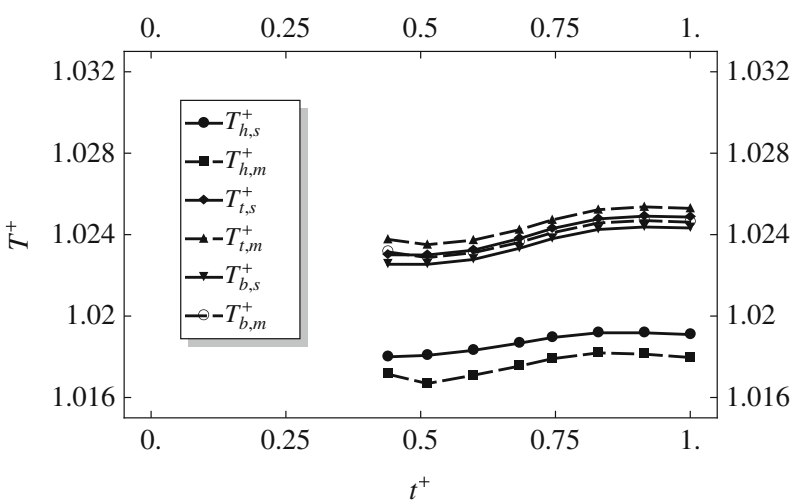


Fig. 15 Dimensionless temperatures-standard vs blocked arm inlets

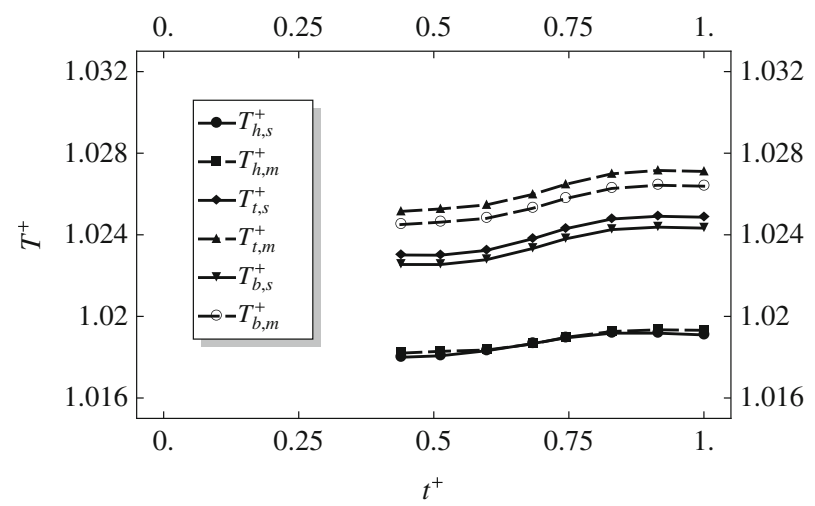

seen from Fig. 14 that the temperature of the head $T_{h, m}$ is lower than $T_{h, s}$ by $0.3-$ $0.4 \mathrm{~K}$ because the cool air stream is higher than in the standard case. This is the positive aspect of this situation. The body temperature $T_{t, m}$ (head excluded) is higher compared to the standard case $T_{t, s}$ since the overall flow of body-directed cooling air decreases because of the blocked leg inlets. This negative aspect dominates the total body temperature $T_{b, m}$ so leading to an overall worse situation than the standard case where the legs and part of the torso are subjected to higher temperatures. For the worker the feeling of thermal discomfort will probably increase. As noted above, this problem may well be common, due to the absolute necessity of using long boots to protect the feet. So these boots tend to block the inlets and reduce the cooling ability of the suit.

When the arm inlets are blocked the whole air stream is divided among the head and leg inlets equally. The head temperature $T_{h, m}$, shown in Fig. 15 is similar to that for the standard case $T_{h, s}$ despite the fact that the local stream of cooling air has increased. The non-head body $T_{t, m}$ temperature and the total body temperature $T_{b, m}$ are significantly higher $(0.7 \mathrm{~K})$ compared to the standard case $T_{t, s}$ and $T_{b, s}$. This situation is even worse than with the leg inlets blocked. This may be because there is in all situations less space around the arms than the legs so that the effect of the

Fig. 16 Dimensionless temperatures - standard vs blocked head inlets

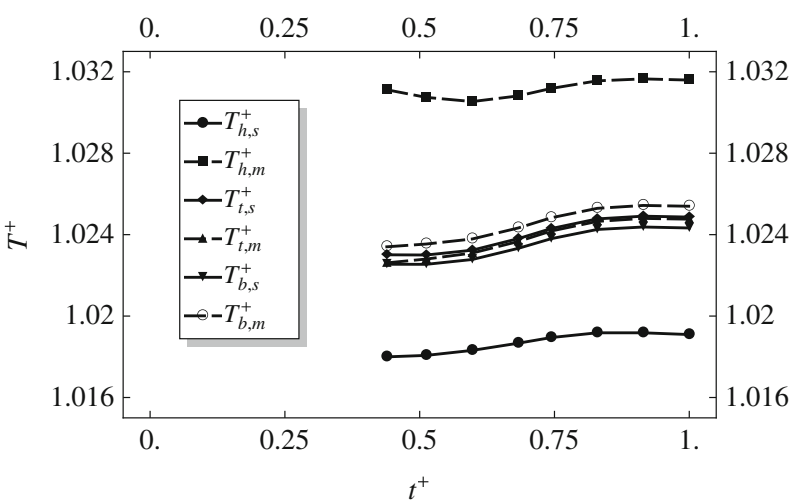


Fig. 17 Dimensionless temperatures - standard vs lower outlets blocked

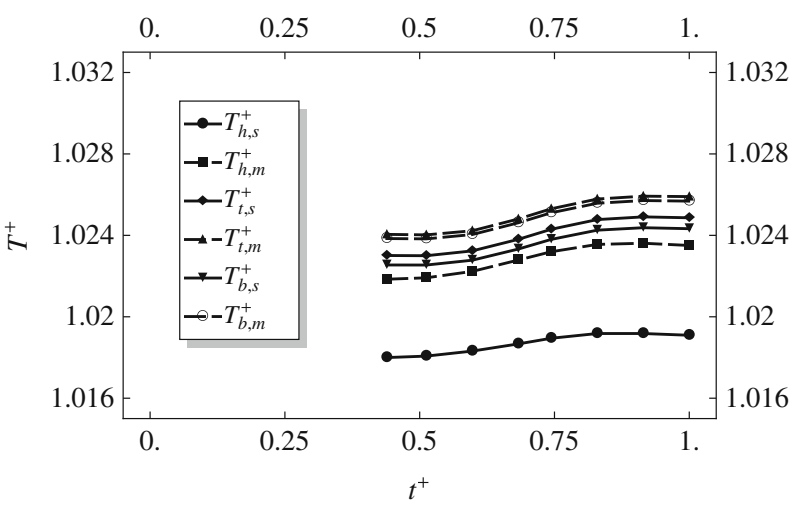

inlet blockage is more marked. Again, it is to be expected that thermal discomfort will increase.

If the head inlets are blocked a significant increase of the head temperature will occur as shown in Fig. 16. The difference between this and the standard case can even exceed $3 \mathrm{~K}$. The situation not only increases thermal discomfort but is dangerous because of the lack of fresh and cool air for breathing. The body temperature, on the other hand, $T_{t, m}$ remains almost the same. However, the total body temperature $T_{b, m}$ is higher compared to the standard case $T_{b, s}$ because of the significant increase in head temperature.

When the lower body outlets are blocked all the air escapes through the upper (head) exits. This means that the length of passage of the cooling air from the lower parts of the suit increases and results in a general temperature increase. In this case all the worker temperatures are higher (whether head $T_{h, m}$, body $T_{t, m}$ or total temperature $T_{b, m}$ ) as shown in Fig. 17. There are no positive aspects of this particular configuration.

Finally, for the case of blocked upper outlets some of the cool air from the head inlets has a longer track length to travel before exiting the suit (rather than immediately disappearing in the upper outlets). This means that greater advantage can be taken of its cooling capability. This situation, therefore, is quite different from

Fig. 18 Dimensionless temperatures - standard vs upper outlets blocked

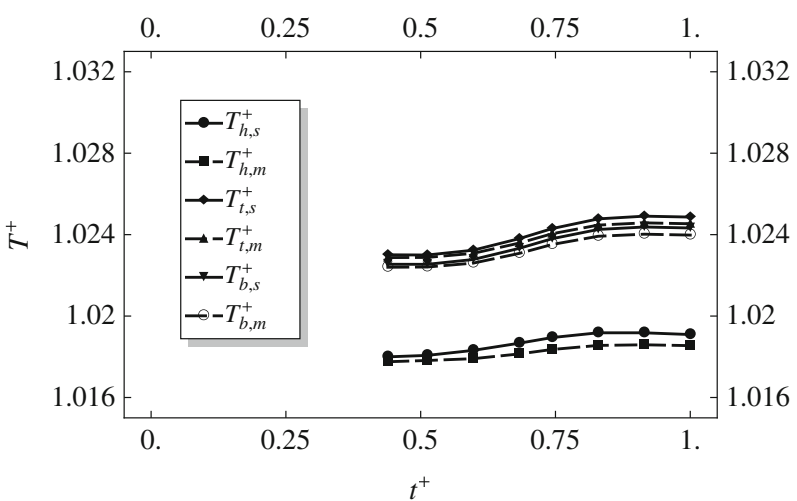


Fig. 19 Modified geometry of pressurised suit

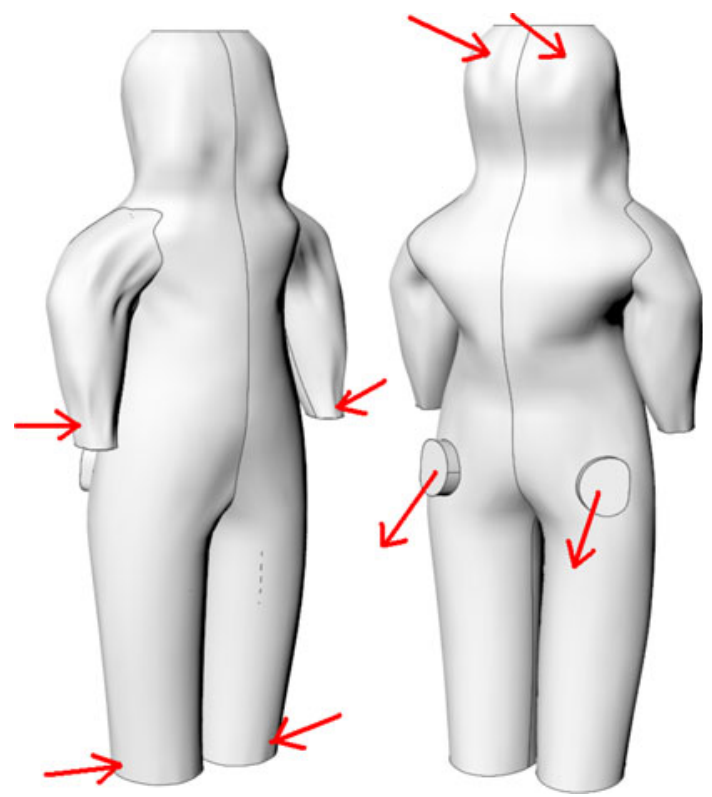

the case of blocked lower outlets where the track length from the head inlet air is significantly shorter. Hence all the temperatures for this case are slightly lower, as shown in Fig. 18.

These analyses of problem cases in fact suggest simple modifications to the original suit configuration which should lead to improvements in performance. In the next section specific suit design changes will be discussed.

\subsection{Improved design}

From the results of the preceding studies on various blockage configurations, it is fairly straightforward to deduce that the original suit design (as in Fig. 5) may be

Fig. 20 Dimensionless temperatures-original vs. modified design

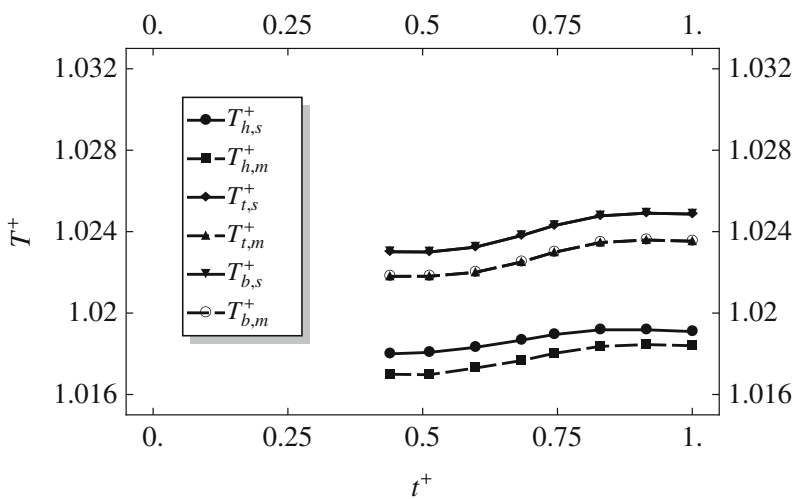




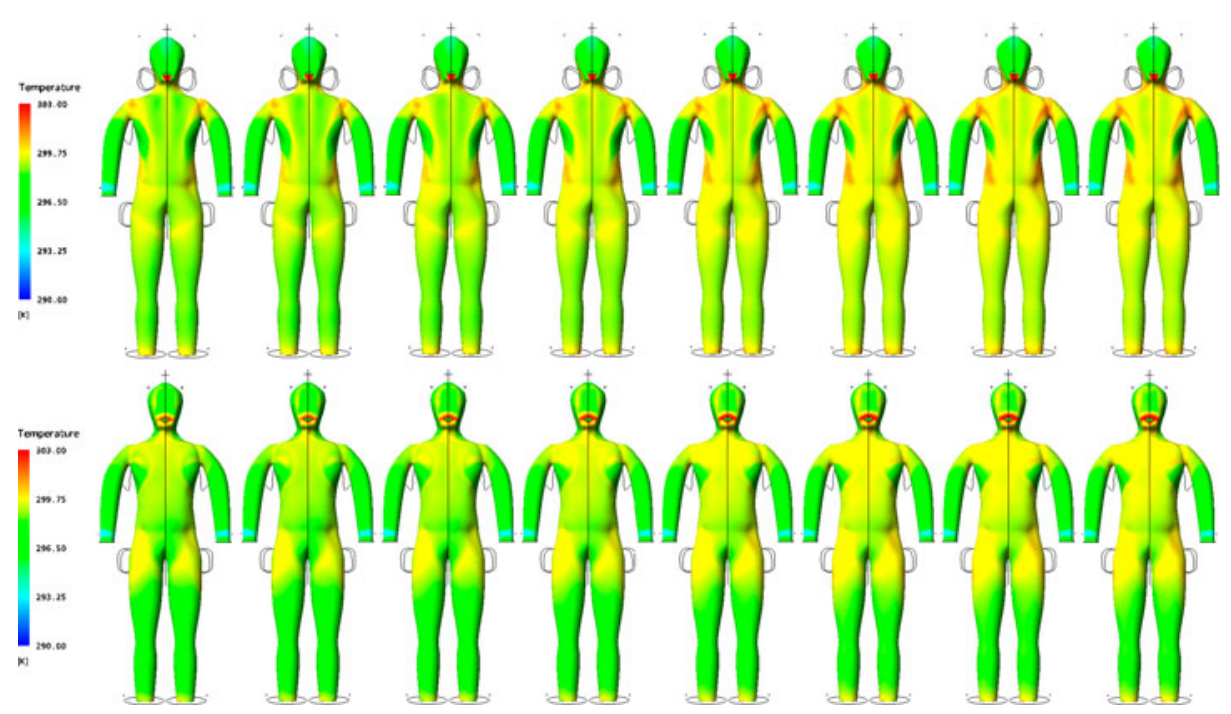

Fig. 21 Body temperature at time step 36, 42, 49, 56, 61, 68, 75 and 82 min-front view. Original (upper) vs modified suit (lower)

changed slightly to obtain lower outlet temperatures and a more uniform distribution of body temperature. Two simple design changes were modeled:

(i) Removal of upper outlets. That redundancy exists in the outlet design is apparent in that the upper outlets are regarded as backup in the case of

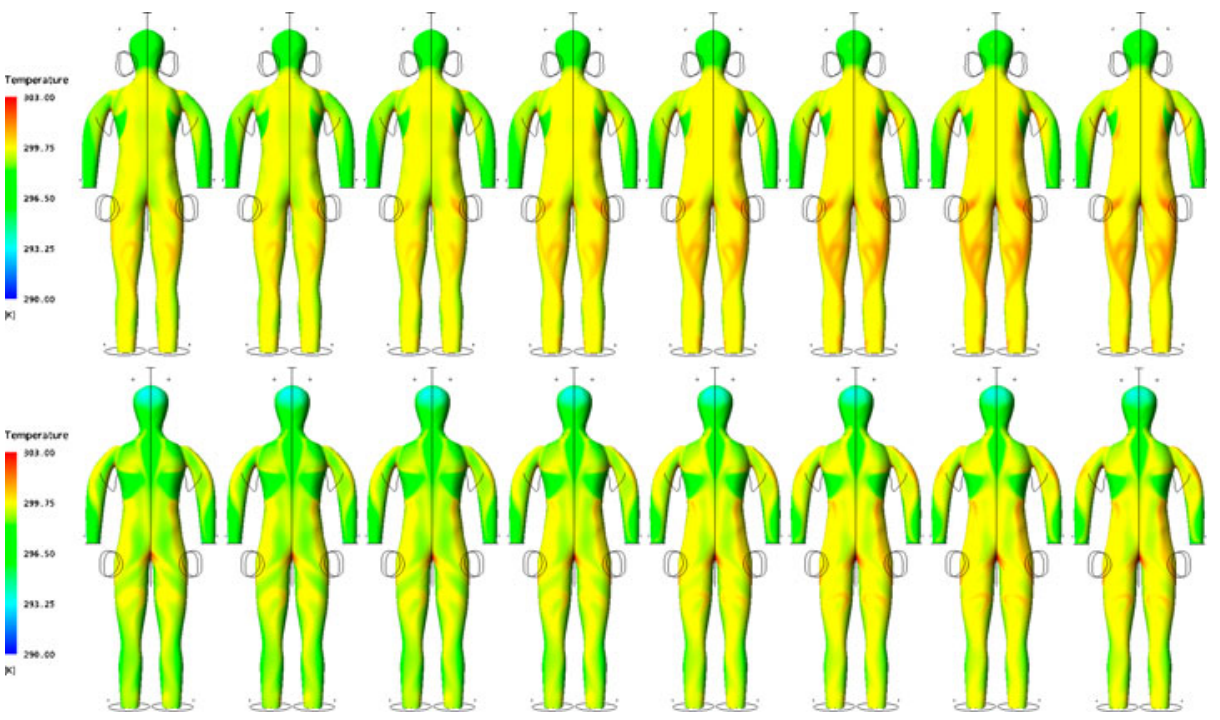

Fig. 22 Body temperature at time step 36, 42, 49, 56, 61, 68, 75 and 82 min-rear view. Original (upper) vs. modified suit (lower) 
blockage of the lower outlets. An alternative design solution to this would be to replace the upper outlets with two more outlets positioned around the already existing lower outlets.

(ii) Repositioning of head inlets at the back of the head rather than on the side, with a downward direction of airflow.

These modifications are relatively simple and should improve the effectiveness of the cooling air. The modified design is shown in Fig. 19. The rest of the design parameters such as mass flow, and its partition among all the inlets, remain the same.

Calculations show that a reduction in temperatures of between $0.1-0.6 \mathrm{~K}$ may be expected over the whole body. These changes are shown in Fig. 20 for the head $T_{h, m}$, body $T_{t, m}$ and the total temperatures $T_{b, m}$. While it is arguable that such temperature reductions are too modest, they are not insubstantial and readily achievable with the proposed modifications, which are both minor and very simple. Figures 21 and 22 demonstrate in detail that most of the hot spots are significantly reduced and the distributions are more uniform.

\section{Summary}

A comprehensive numerical and computational model has been developed to treat the thermo-fluids of the microcirculation between the human body surface and the interior of a whole-body pressurised suit as used in the JET fusion reactor. The overall model uses commercial CFD software as a basis and includes a carefullyderived additional two-component model to allow for the humidity aspect of thermal comfort. The geometries of the suit and its wearer were fully three-dimensional and reconstructed from a high-accuracy industrial-scale experiment using a mannequin with and without a suit. The experiment used photographic scanning, which could also accommodate simulated transients involving worker's movements. Validation experiments with a suited (human) worker involved measurements of cooling air temperature rises related to the worker's metabolic levels. The model was then used to test proposals for improving the thermal performance of the suits. The hotspots on the body were significantly reduced and the distribution of the temperature was more uniform when the inlets and outlets of the cooling air were repositioned. This shows the potential of the overall comprehensive model in permitting relatively easy checks for various configurations without the need for manufacturing a range of test suits. While this does not make measurement and validation unnecessary it does save considerable time, effort and expense for design changes. The generic nature of the modelling means it could be applied to other suit designs for other purposes.

Acknowledgements This project is funded by the Engineering and Physical Sciences Research Council and the UK Atomic Energy Authority.

Open Access This article is distributed under the terms of the Creative Commons Attribution Noncommercial License which permits any noncommercial use, distribution, and reproduction in any medium, provided the original author(s) and source are credited. 


\section{References}

1. Bishop, P., Ray, P., Reneau, P.: A review of the ergonomics of work in the us military chemical protective clothing. Int. J. Ind. Ergon. 15(4), 271-283 (1995)

2. Edwards, P., Tesch, K., Karayiannis, T.G., Collins, M.W., Atherton, M.A., Young, P., Large, M., Brade, A.: Design optimisation of air-fed full pressurised suits. Fusion Eng. Des. 84(2-6), 716-721 (2009)

3. Fan, J., Chen, Y.: Measurement of clothing thermal insulation and moisture vapour resistance using a novel perspiring fabric thermal manikin. Meas. Sci. Technol. 13(7), 1115-1123 (2002)

4. Ferziger, J.H., Perić, M.: Computational Methods for Fluid Dynamics. Springer (2002)

5. Gonzalez, R., McLellan, T., Withey, W., Chang, S., Pandolf, K.: Heat strain models applicable for protective clothing systems: comparison of core temperature response. J. Appl. Physiol. 83(3), 1017-1032 (1997)

6. Henane, R., Bittel, J.: Changes of thermal balance induced by passive heating in resting man. J. Appl. Physiol. 38(2), 294-299 (1975)

7. Havenith, G.: Heat balance when wearing protective clothing. Ann. Occup. Hyg. 43(5), 289-296 (1999)

8. Ishii, M., Hibiki, T.: Thermo-Fluid Dynamics of Two-Phase Flow. Springer (2006)

9. Jones, W., Launder, B.: The prediction of laminarization wit a two-equation model of turbulence. Int. J. Heat Mass Transfer 15(2), 301-314 (1972)

10. Kolev, N.I.: Multiphase Flow Dynamics, vols. 1, 2. Springer (2005)

11. Menter, F.R.: Two-equations eddy-viscosity turbulence models for engineering applications. AIAA J. 32(8), 1598-1605 (1994)

12. Nigmatulin, R.: Dynamics of Multiphase Media, vol. 1. Taylor \& Francis (1990)

13. Peppers, V.: ASHRAE Handbook Fundamentals, ASHRAE, pp. 6.9-6.10 (1997)

14. Tesch, K., Atherton, M.A., Karayiannis, T.G., Collins, M.W., Edwards, P.: Heat transfer coefficient calibrations by means of evolutionary algorithms. In: The $8^{\text {th }}$ International Conference on Adaptive Computing in Design and Manufacture (ACDM 2008), Bristol, U.K. (2008)

15. Tesch, K., Karayiannis, T.G., Collins, M.W., Atherton, M.A., Edwards, P.: Thermo-fluid transport phenomena in air-fed pressurised protective clothing. In: 5th International Conference on Transport Phenomena in Multiphase Systems (HEAT 2008), Bialystok, Poland (2008)

16. Tesch, K., Collins, M.W., Karayiannis, T.G., Atherton, M.A., Edwards, P.: A Thermo-Fluid Model for Protective Suiting Used in Fusion Reactor Shutdown Operations. XXVI Congresso Nazionale UIT sulla Trasmissione del Calore, Palermo, Italia (2008)

17. Tesch, K., Collins, M.W., Karayiannis, T.G., Atherton, M.A., Edwards, P.: Heat and mass transfer in air-fed pressurised suits. Appl. Therm. Eng. 29(7), 1375-1382 (2008)

18. Tesch, K., Atherton, M.A., Karayiannis, T.G., Collins, M.W., Edwards, P.: Determining heat transfer coefficients using evolutionary alogrithms. Eng. Optim. 41(9), 855-870 (2009)

19. Wilcox, D.C.: Turbulence Modeling for CFD. DCW Industries (1994)

20. Yakhot, V., Orszag, S., Thangam, S., Gatski, T., Speziale, C.: Development of turbulence models for shear flows by a double expansion technique. Phys. Fluids 4(7), 1510-1520 (1992) 